\title{
DEVELOPMENT OF LED LIGHT SOURCES IN LANDSCAPE LIGHTING
}

\author{
Zhiqiao WANG \\ School of Art Yangtze University, Jingzhou Hubei 434000, China; \\ E-mail: zhangque8@yeah.net
}

\begin{abstract}
An LED light source, which is extensively used in landscape design, has played a good role in its application due to its characteristics and advantages. On the basis of cost-effectiveness analysis, green lighting energy-saving technologies that use LED light sources in landscapes are analyzed, as well as the key factors that affect the cost-effectiveness of various stakeholders. Results show that the cost-effectiveness analysis method is effective for evaluating the DSM technology. Moreover, the application of LED light source in landscape lighting has great feasibility. This study provides some theoretical reference for the promotion of green lighting energy-saving technology.
\end{abstract}

Keywords: LED, illumination, cost-effectiveness, DSM technology, cluster analysis method, analytic hierarchy process (ANP)

\section{INTRODUCTION}

By 2050, approximately $75 \%$ of the world's population will be living in urbanized areas. Urbanization leads to energy crisis and environmental issues, as well as lifestyle changes. These trends cause the lighting industry to undergo a major transformation. The rapid development of LEDs has made this revolution possible not only because the energy-saving characteristic of LED lighting meets the requirements of the form; moreover, the dynamic changes not only further suit the needs of the market but also able to render the lights under night conditions [1]. Garden landscapes are ideal paradise and motivation for people. A garden fully embodies people's yearning and desire for a free life. It is a concentrated expression of aesthetic cognition and thinking applied by humans in the transformation of nature and the use of natural processes to create a living environment [2]. Landscape stones are typically used for hard landscape. Although these stones have dull appearance, they seem to emanate life after artificial selection and stacking, such that they look natural. These stones ingeniously imply the natural and philosophical views of "harmony between man and nature." Strong and weak light changes, colour matching, and strong irradiation are fully used in garden lighting, which increases the brilliance of garden flowers, trees, and landscape buildings at night, thereby fully expressing the characteristics of night scenes and fully displaying the design style of an architecture or landscape to achieve elegant lighting atmosphere [3]. Lighting designers often use several urban architectural and square lighting methods to redesign traditional gardens, such that peaceful and distant natural garden landscape becomes distraught, noisy, and exceedingly bright and modern gardens are always full of sound and light effects [4].

\section{STATE OF THE ART}

Garden lighting designs should have specific use of lights to ensure that distinctive buildings, sculptures, flowers, rocks, and mountains have different appearance during daytime. A quiet and tranquil atmosphere is present in the light and shadow created by light. However, nightscape lighting designs should meet a series of ecological and psychological requirements to avoid problems, such as 
glare and light pollution. Night scene lighting must meet the professional and artistic fusion to ensure that political and cultural qualities, artistic heritage, and historical background of a city or township are appropriately reflected. Meanwhile, the lighting design should express a rich landscape culture and reflect its cultural heritage [5]. China has no noticeable development trend of garden lightings until the 1990s. The subject of lighting that time was buildings, and garden landscapes only needed basic lighting functions. Moreover, landscape lighting is only utilized during festivals. The lighting was specifically used for a short time and its method was simple. Simple objects mostly used contour and floodlighting. With the active development of science and technology, garden landscapes have gradually expanded to its full range [6]. Lighting techniques and designs have also become increasingly complex and diverse. From the use of a single layer to the utilization of integrated light sources, the three-dimensional collocation of various lighting fixtures has enabled the current variety of lighting technologies to emerge in an endless stream. "Green lighting” is a new lighting approach proposed after the 1990s on the basis of the perspective of energy conservation and environmental protection [7].

\section{METHODOLOGY}

\subsection{Establishment of LED Lighting Indicator System and Determination of Indicator Weight}

The emergence of white LED at the end of the $20^{\text {th }}$ century has attracted widespread attention. It is the fourth generation of light source for the purpose of green lighting. With the continuous advancement of LED technology in recent years, LED light sources are gradually replacing traditional light sources in urban landscape lighting. Hence, LED lights are increasingly favoured by designers because of its advantages, such as good concealment, low power, concentrated light, and controllability [8]. However, thus far, LED light source has no evident advantage over traditional light sources in several areas, such as road and hotel interior lightings; hence, people must not rapidly adopt this technology. In recent years, designers have continuously conducted theoretical research on the lighting of garden sketches. Researchers extensively use plants as examples to investigate their changes in light and shadow. However, research on this subject is relatively rare.
In the cluster analysis method, we must clearly classify the criteria and weights. First, we need to establish a judgment matrix that represents the aforementioned level as a benchmark. Then, we compare the relative importance of the two-level indicators. Judgment matrix A is adopted in this study, where $a_{i j}=1 / a_{j i}$ and $a_{i i}=1,1 \leq i \leq j \leq n$. The matrix weights and the product $M_{i}$ of the elements of each row of judgment matrix A are calculated as follows:

$$
M_{i}=\prod_{j=1}^{n} a_{i j}, i=1,2,3,4 \ldots n .
$$

The $n^{\text {th }}$ root value of each row $M_{i}$ is calculated as

$$
\bar{W}_{2}=\sqrt{M_{2}}, i=1,2,3,4 \ldots n .
$$

Vector $\left(\bar{W}_{1}, \bar{W}_{2}, \ldots \bar{W}_{N}\right)^{T}$ is normalized, and $W_{i}$ denotes the weight coefficient value of each obtained index, as expressed as follows:

$$
W_{i}=\frac{\bar{W}_{i}}{\sum_{j=1}^{n} \bar{W}_{j}} .
$$

Then, consistency check is performed. Practically, the judgment matrix should satisfy the general consistency, and consistency check is required. We can prove that the judgment matrix is logically reasonable to continue analyzing the results only through inspection. The maximum eigenvalue is calculated as

$$
\lambda_{\max }=\sum_{i=1}^{n} \frac{(A W)_{i}}{n W_{i}} .
$$

Then, the consistency index $C I$ is calculated as

$$
C I=\frac{\lambda_{\max }-n}{n-1} .
$$

The corresponding average random consistency index $R I$ is obtained on the basis of the difference of various orders of the judgment matrix, as shown in Table 1.

Table 1. An Average Random Consistency Index RI

\begin{tabular}{|c|c|c|c|c|c|c|c|c|c|}
\hline $\mathrm{n}$ & 1 & 2 & 3 & 4 & 5 & 6 & 7 & 8 & 9 \\
\hline $\mathrm{RI}$ & 0 & 0 & 0.58 & 0.89 & 1.12 & 1.26 & 1.36 & 1.41 & 1.46 \\
\hline
\end{tabular}

First, the consistency ratio $C R$ is calculated and analyzed. The consistency of the judgment matrix is 
considered acceptable when $C R<0.1$. Meanwhile, when $C R>0.1$, the consistency requirement is not met; hence, the judgment matrix must be revised. Finally, the index system weight determination and consistency check are performed. Three criteria indicators, namely, consumption, profit, and user indicators of the mobile environment are set under the consumer value evaluation index. The result corresponded with the matrix $A_{1}$ after comparing the first-class indicators by using the available questionnaire number.

$$
A_{1}=\left[\begin{array}{ccc}
1 & 3 & \frac{1}{3} \\
\frac{1}{3} & 1 & \frac{1}{5} \\
3 & 5 & 1
\end{array}\right] .
$$

A step-by-step calculation can be performed to obtain each index. In addition, we interviewed nine experts in related fields to establish a mobile consumer segmentation model. After calculating the weights of the indicators provided by each expert, the weights of the final indicators are identified using the arithmetic average method. The statistics of the final results of the first-level indicators of value evaluation are not listed due to the limited space. Table 2 shows the final weight of the analytic hierarchy process (AHP).

We use AHP to analyze and compare the competitiveness of Jiangmen with the developed cities of the LED industry, such as Shenzhen, Dongguan, Zhongshan, and Foshan in the Pearl River Delta.
Therefore, the indicators are transformed into a hierarchical structure (Fig. 1).

\subsection{Lighting Cost-Effectiveness Analysis}

The cost-effectiveness of lighting is analyzed. During user cost analysis, users participate in the LED green lighting energy-saving project to pay the additional cost by replacing incandescent lamps with energy-saving lighting products, that is, direct costs or increased equipment purchase fees, which can be expressed as

$$
C c=\sum_{j \in J, k \in K}\left(C_{c(j, k)} n_{(j, k)} N_{(j, k)}\right),
$$

where

$$
C_{c(j, k)}=\left(P_{j}-Q_{j} / Q_{b} P_{b}\right) /\left(p_{b}-p_{j}\right),
$$

where $C_{c}$ is the total direct cost of the user in yuan/W, $k$ is the collection of user categories (such as residents, shopping malls, hotels, and restaurants) participating in the green lighting energy-saving project, and $j$ is the collection of green lighting products. At present, a large number of mature and simple lighting energy-saving measures use compact fluorescent lamps (commonly known as energy-saving lamps) instead of incandescent lamps or electronic ballasts instead of ordinary magnetic ballasts for fluorescent lighting. $C_{c(j, k)}$ is the direct cost of the use of the seventh type green lighting product for the $k^{\text {th }}$ user to save $1 \mathrm{~W}$ electricity, yuan/W; and $N_{(j, k)}$ is the number of users who are interested in replacing the original lighting products with the seventh type of green lighting products. The corpse and

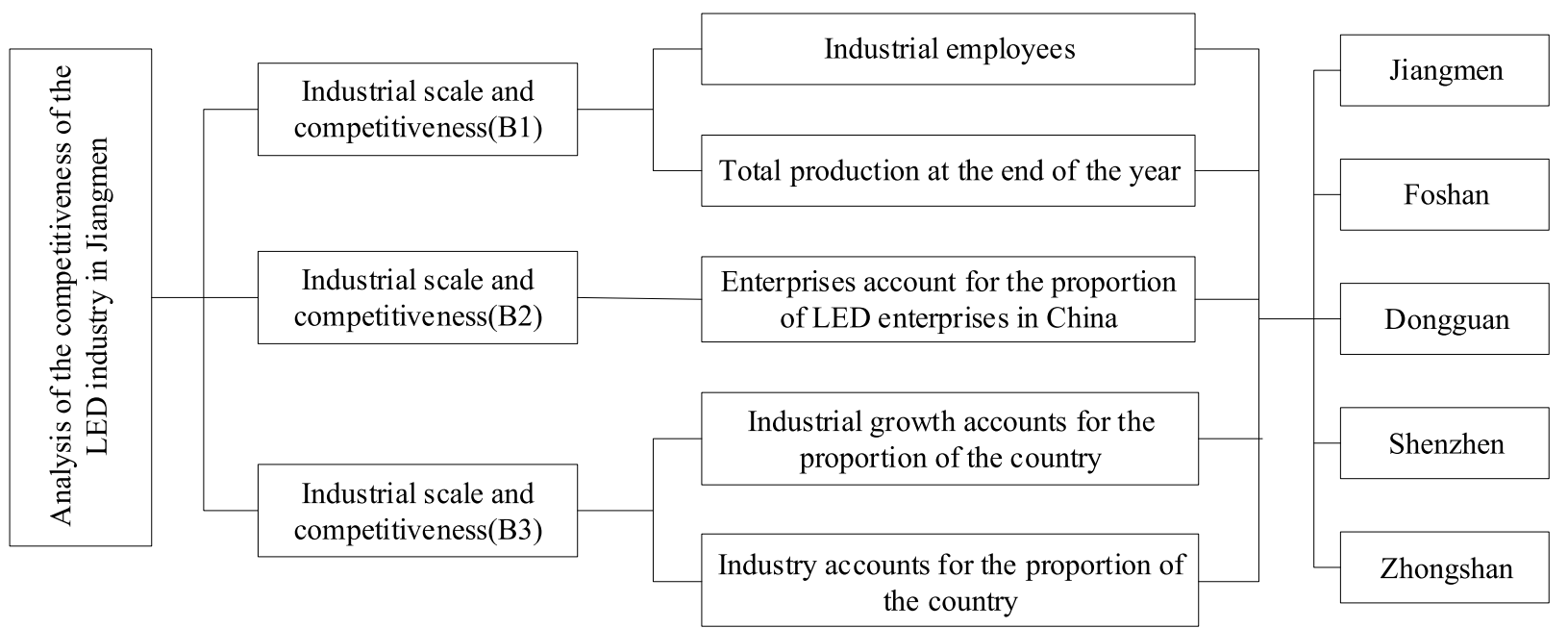

Fig.1. Hierarchical structure diagram 


\section{Table 2. Indicators for the Comprehensive Competitiveness Evaluation System of the LED Industry in Jiangmen}

\begin{tabular}{|c|c|c|}
\hline \multirow{10}{*}{$\begin{array}{l}\text { The comprehensive } \\
\text { competitiveness of } \\
\text { the LED industry in } \\
\text { Jiangmen }\end{array}$} & First level index & Two level index \\
\hline & \multirow{3}{*}{$\begin{array}{l}\text { Industrial scale and } \\
\text { competitiveness } \\
\text { Industrial growth competitiveness } \\
\text { Competitiveness of industrial } \\
\text { market }\end{array}$} & Number of industrial employees (only) \\
\hline & & Total production at the end of the year (billion yuan) \\
\hline & & Industrial GDP at the end of the year (billion yuan) \\
\hline & \multirow{3}{*}{$\begin{array}{l}\text { First level index } \\
\text { Industrial scale and } \\
\text { competitiveness } \\
\text { Industrial growth competitiveness }\end{array}$} & $\begin{array}{l}\text { Average growth rate of industrial value in the past } \\
\text { three years }\end{array}$ \\
\hline & & $\begin{array}{l}\text { Enterprises account for the proportion of LED en- } \\
\text { terprises in China (cy0) }\end{array}$ \\
\hline & & $\begin{array}{l}\text { Industry production accounts for the proportion of } \\
\text { national output (\%) }\end{array}$ \\
\hline & \multirow{3}{*}{$\begin{array}{l}\text { Competitiveness of industrial } \\
\text { market }\end{array}$} & $\begin{array}{l}\text { Industrial output accounts for the proportion of } \\
\text { LED industry in China }\end{array}$ \\
\hline & & $\begin{array}{l}\text { Industrial growth accounts for LED growth in the } \\
\text { country (\%) }\end{array}$ \\
\hline & & $\begin{array}{l}\text { The export value of the industry accounts for the } \\
\text { proportion of the export value of the region (\%) }\end{array}$ \\
\hline
\end{tabular}

cadaver are the market prices of the original lighting products and the seventh type of green lighting products, respectively. $Q_{b}$ and $Q_{j}$ are the average life of the original lighting products and the seventh type of green lighting products, $h$, respectively. $P_{b}$ and $P_{j}$ are the power of the original lighting products and the seventh type of green lighting products, in W, respectively. In addition, $N_{0}$ is the total number of users in a certain area, and $i_{(j, k)}$ is the participation rate of the $k^{\text {th }}$ user who is willing to replace the original lighting product with the $j^{\text {th }}$ green lighting product. In the user benefit analysis, the benefit accumulated by users through the use of energy-saving lighting products is the energy-saving benefit during the product life cycle accumulated by all the different types of users participating in the green lighting energy-saving project. The power-saving benefits that users receive during the life cycle of green energy saving lighting products are as follows:

$$
B_{c(j, k)}=\Delta W_{c(j, k)} P_{k},
$$

where

$$
\Delta W_{c(j, k)}=\left(p_{b}-p_{j}\right) Q_{j} n_{(j, k)} N_{(i, k)} \times 10^{-3},
$$

$\mathrm{B}_{\mathrm{c}(j, k)}$ is the power-saving benefit obtained by the $k^{\text {th }}$ user through the use of the seventh type of green lighting products; $\Delta W_{c(j, k)}$ is the amount of electricity used by the $k^{\text {th }}$ class user for the $j^{\text {th }}$ green lighting product, $\mathrm{kW} \cdot \mathrm{h}$; and $P_{k}$ is the lighting power consumption of the $k^{\text {th }}$ user, yuan $/(\mathrm{kW} \cdot \mathrm{h})$. The static investment recovery period of the users who utilize various types of energy-saving technologies is one of the key factors that affect the willingness of users to participate in the DSM measure. The static investment payback period for users who utilize green lighting products is expressed as

$$
T_{j}=\frac{P_{j}-Q_{j} / Q_{b} P_{b}}{P_{k} h_{j}\left(p_{b}-p_{j}\right) \times 10^{-3}}=\frac{C_{c(k, j)}}{P_{k} h_{j} \times 10^{-3}},
$$

where $h_{j}$ is the daily illumination time of the seventh type of green lighting product, $\mathrm{h} / \mathrm{d}$.

\subsection{Association Rule Algorithm}

The Apriori algorithm mines item sets through constant construction and filtration. Each screening requires a scan of all the raw data. Therefore, scanning the original data multiple times is necessary. When the size of the original data is large, the efficiency is relatively low. Thus, scholars have found ways to reduce the number of scans of the original data, simplify the strategy of mining frequent 
Table 3. Average Random Consistency Index R (1000 Positive Reciprocal Matrix Calculation Results)

\begin{tabular}{|c|c|c|c|c|c|c|c|c|}
\hline Matrix order & 1 & 2 & 3 & 4 & 5 & 6 & 7 & 8 \\
\hline R.I. & 0 & 0 & 0.52 & 0.89 & 1.12 & 1.26 & 1.36 & 1.41 \\
\hline Matrix order & 9 & 10 & 11 & 12 & 13 & 14 & 14 & 15 \\
\hline R.I. & 1.46 & 1.49 & 1.52 & 1.54 & 1.56 & 1.58 & 1.58 & 1.59 \\
\hline
\end{tabular}

item sets, simplify calculations, and shorten the time consumption. To avoid the defects of the Apriori algorithm, Han et al. proposed the frequent pattern (FP)-growth algorithm. This algorithm does not generate candidate frequent item sets but only scans the original data twice. Compression of raw data through FP-tree data structure is increasingly efficient. Therefore, the FP-growth algorithm is an efficient algorithm for mining frequent item sets in the case of large data size.

The FP-growth aims to construct an FP-tree with frequent item sets to retain the item set information during the first pass scan. Then, the FP-tree is divided into several conditional libraries, which are then mined. The FP-growth algorithm mainly involves two steps, namely, FP-tree construction and recursive mining. The FP-tree construction compresses the transactions in the original data to an FP-tree via two data scans. The FP-tree is similar to a prefix tree, and the paths of the same prefix can be shared to compress the data. Then, the FP-tree is used to identify the conditional pattern base and FPtree of each items, and the recursive mining condition of the FP-tree acquires all the frequent item sets. Similarly, we use the examples provided previously to describe the main steps of the FP-growth algorithm in detail.

The FP-tree is initially constructed. The entire database is scanned; hence, the frequent item sets and their support for a single item is $\{a\}: 5,\{b\}: 4,\{c\}: 6,\{d\}: 7,\{e\}: 1$.

The minimum support number is set to 1 , and the result is presented in descending order as $\mathrm{A}=$ $\{\{d\},\{c\},\{a\},\{b\}\}$ S. Hence, the FP-tree can be constructed. First, an empty root node is established. Then, the database is scanned for the second time. Finally, a branch for each order is created in accordance with the previous support order. For example, $\mathrm{a}, \mathrm{d}$, and e are present in Order No. 1. Hence, the order is three branches $d(7)-a(5)$ according to the support degree. The empty node is connected to d, which is connected to a; the branch node corresponding to Order No. 2 is d(7)-b(4). Meanwhile, a header table (Header Table) is created. The project, its support, and its nodes are recorded to facilitate traversal in the subsequent mining. The constructed FP-tree and item header table are shown in Fig. 2.

Then, the FP-tree is recursively mined.

\section{RESULT ANALYSIS AND DISCUSSION}

Simulation experiments are conducted through the BP neural network to design the most energy efficient lighting installation scheme that satisfies the illumination standard value. Generally, the lighting fixture installation position and power in different environments will have a certain lighting effect.

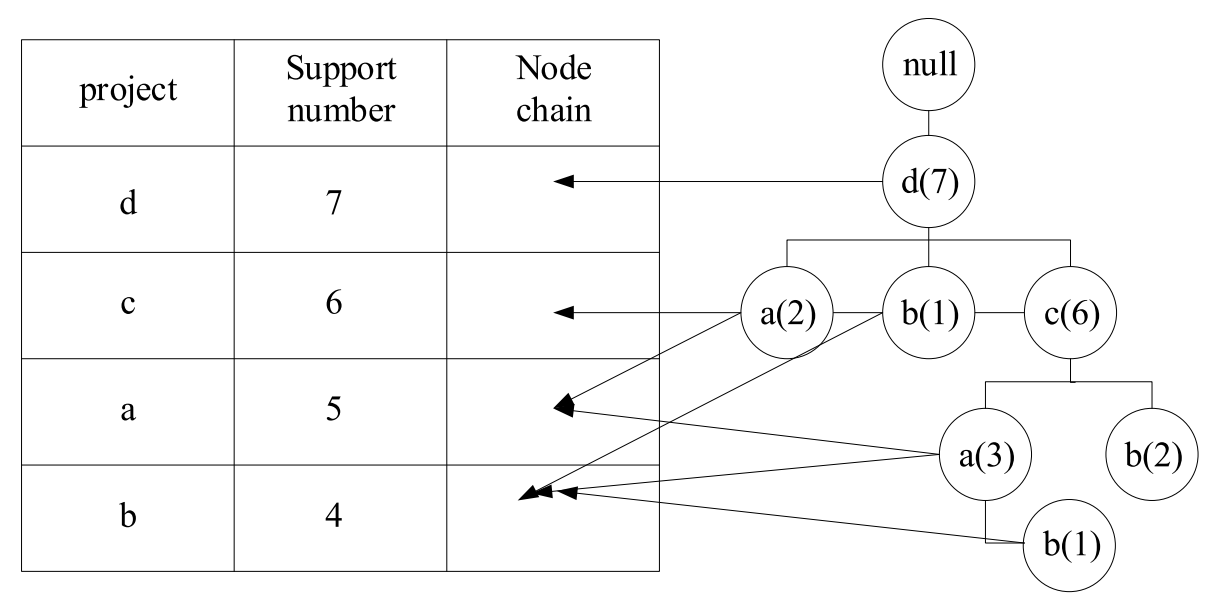

Fig.2. Header Table and FP-tree 


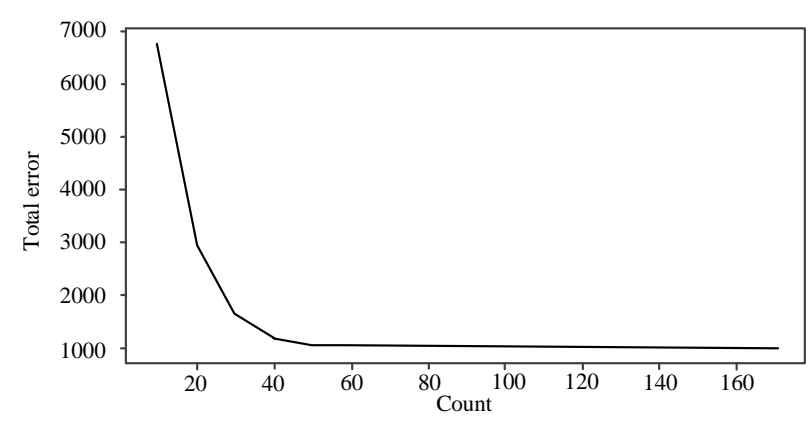

Fig.3. BP neural network algorithm (a)

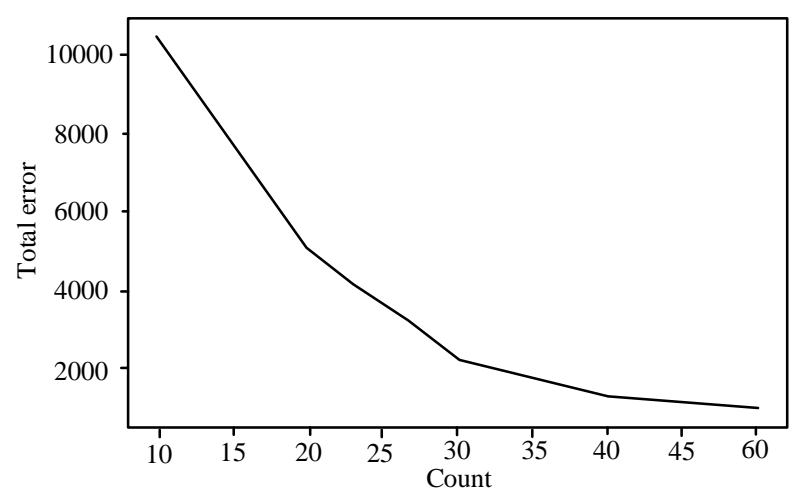

Fig.5. BP neural network algorithm (c)

A model is established using the BP neural network, and the illuminance standard and the luminaire power are defined as the input quantities. Furthermore, the installation position of the luminaire is defined as the output quantity, and the training is performed. We select a classroom in as an experimental object. The input data of the BP algorithm is normalized, the input and output parameters of the network are determined, and the selection and determination of the parameters are selected according to actual conditions. The data used in the test show that the input quantities are the lighting brightness and the lamp power, whereas the output are the minimum horizontal and vertical spacing (in $\mathrm{cm}$ ) of the lamps installed in the selected classroom. The default size of the classroom is $12 \mathrm{~m} \times 9$ $\mathrm{m}$. Evaluations can be performed on data modified for classrooms of various sizes. During the test, fluorescent lamps are used as test fixtures. The $16 \mathrm{~W}$ LED lamp used for this design is generally the same as the fluorescent lamp $40 \mathrm{~W}$ in illumination. Thus, the simulation of the $40 \mathrm{~W}$ lamps can be the basis for the installation position of the lamp in the design. The total error of the training obtained after running multiple times is shown in Figs. 3, 4, 5. The error of the algorithm is graphically displayed every 10 iterations.

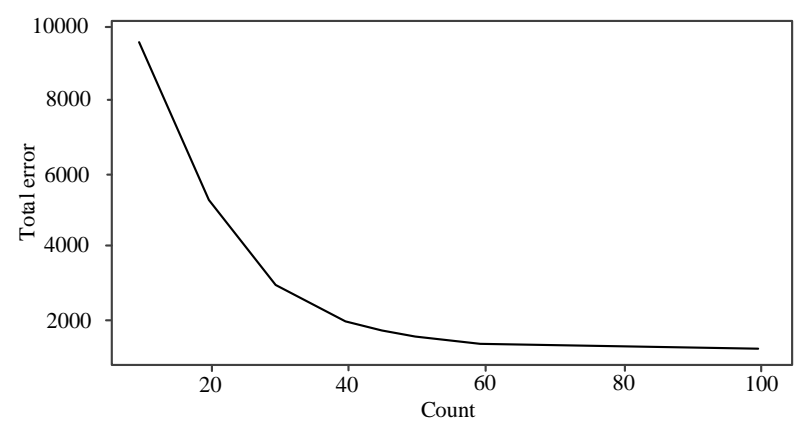

Fig.4. BP neural network algorithm (b)

The condition pattern base for each item is created starting with the last item (lowest support) in the header table. All paths that contain the project are calculated, and the project's prefix path is the conditional mode base. For example, if the path of $b$ item is $d-b, c-a-b, c-b, d-c-b$, and b, then the prefix path is $d, d-c-a, c$, and $d-c$, that is, the condition mode base of item $b$. Then, the condition tree of the project is constructed using the set of conditional schema bases of the project with minimum support. All combinations of frequent item sets are generated on the basis of the condition tree. Let the minimum support number be 1 . For $b$, the resulting frequent item set is $\{b, d\}\{b, c\}$. The numbers of support for these frequent item sets are 2 and 3 . The results obtained through the Apriori algorithm are the same. However, the FP-growth algorithm has its disadvantages. Given that the algorithm recursively generates conditional databases and FPtrees, it consumes a large amount of memory. This large memory consumption is the main problem of this algorithm. The advantages of the recommendation based on association rules are its simplicity and perceptivity; moreover, it can imitate an actual shopping scene and does not need knowledge related to the product while possibly exploring the potential interests of users and purchase stimulations. However, computational complexity is a considerable drawback of association rule mining. With the increase in the size of frequent item sets, especially in the case of large data sets, the cost of mining will also increase. Nevertheless, given the same minimum support, confidence, and other constraints for the same data set, the association rules obtained by the Apriori and FP-growth algorithms are exactly the same.

Finally, the test results are obtained, which can be used to meet the illuminance requirement if a $40 \mathrm{~W}$ lamp is selected in a $12 \mathrm{~m} \times 9 \mathrm{~m}$ classroom. Five and six fixtures can be installed in the $\mathrm{X}$ - and 
Y-axis ranges, respectively. The entire classroom requires 30 lamps.

\section{CONCLUSION}

At present, lighting designers often use several urban architectural lighting and square lighting methods in designing traditional gardens and construct garden landscapes. Traditional gardens are originally peaceful, distant, and natural but become distraught, noisy, and saturated with light. Garden landscapes are consistently full of modern gardens with sound and light effects. Hence, relevant data in the overall design of the system are reviewed in this study. Then, the current status in the development of the green lighting technology of LED light source and its advantages, composition, and development overview are evaluated. The existing LED light source green lighting systems are analyzed inside and outside the country. The overall design ideas and programs are formed in combination with the current condition of landscape lighting in China. The cost-effectiveness of the green lighting energy-saving technology is investigated on the basis of the cost-effectiveness method by using LED light sources in the landscape. The method provides a theoretical basis and technical guidance for the implementation of the green lighting project. The results show that the cost-effectiveness analysis method is effective for evaluating the DSM technology. The method can also be applied to other DSM measures. Therefore, conducting a quantitative cost-effectiveness study is worthwhile to evaluate the feasibility of a certain DSM measure.

\section{REFERENCES}

1. Farahat A, Florea A, Lastra J L M, et al. Energy efficiency considerations for LED-based lighting of multipurpose outdoor environments. IEEE Journal of Emerging and Selected Topics in Power Electronics, 2015. V3, \#3, pp.599-608.

2. Leccese F. Remote-control system of high efficiency and intelligent street lighting using a ZigBee network of devices and sensors. IEEE transactions on power delivery, 2013. V28, \#1, pp.21-28.

3. Xu, X., Xie, L., Li, H., \& Qin, L. Learning the route choice behaviour of subway passengers from AFC data. Expert Systems with Applications, 2018. \#95, pp. 324-332.

4. Basker D K, Cortes O A H, Brook M A, et al. 3D Nonlinear Inscription of Complex Microcomponents (3D NSCRIPT): Printing Functional Dielectric and Metallodielectric Polymer Structures with Nonlinear Waves of Blue LED Light. Advanced Materials Technologies, 2017. V2, \#5, p.1600236.

5. Haiying Li, Xian Li, Xinyue Xu, Jun Liu, Bin Ran. Modeling departure time choice of metro passengers with a smart corrected mixed logit model - A case study in Beijing. Transport Policy. 2018, \#69, pp.106-121.

6. Pimputkar S, Speck J S, DenBaars S P, et al. Prospects for LED lighting. Nature photonics, 2009. V3, \#4, p.180.

7. De Rossi F, Pontecorvo T, Brown T M. Characterization of photovoltaic devices for indoor light harvesting and customization of flexible dye solar cells to deliver superior efficiency under artificial lighting. Applied Energy, 2015. V156, pp.413-422.

8. Wang L, Wang X, Kohsei T, et al. Highly efficient narrow-band green and red phosphors enabling wider color-gamut LED backlight for more brilliant displays. Optics Express, 2015. V23, \#22, pp.28707-28717.

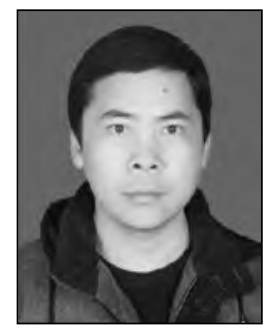

\section{Zhiqiao WANG}

received his Master Degree from Wuhan University in 2012, and received his bachelor degree from Huazhong University of Science \& Technology in 2004. Now, he is working as a lecturer in Yangtze University. His current research interests include digital art design, landscape design and interior design 